\title{
Impacts du coton-Bt sur les bilans financiers des sociétés cotonnières et des paysans au Burkina Faso
}

\author{
Michel Fok ${ }^{*}$ \\ CIRAD, UPR AIDA, avenue Agropolis, 34398 Montpellier cedex 05, France
}

\begin{abstract}
Résumé - Depuis 2008, le Burkina Faso a adopté le coton-Bt en milieu paysan, à partir de variétés africaines transformées. L'étude menée est la première étude ex post, couvrant la période de 2008-2013, relative aux impacts financiers respectifs des sociétés cotonnières et des paysans, chaque catégorie étant prise globalement. L'étude est originale en tenant compte de la transformation génétique imparfaite des variétés utilisées. L'étude évalue les impacts financiers d'une situation réelle d'utilisation du coton-Bt par rapport à une situation de référence sans recours au coton-Bt. L'évaluation est faite aussi par rapport au scénario anticipé lors de la décision d'adoption et dont l'hypothèse d'un gain de rendement au champ de $30 \%$ n'a pas été confirmée. L'évaluation est réalisée par la méthode de budget partiel avec des calculs de sensibilité à trois critères, industriel, commercial et du prix mondial. Pour les paysans, l'impact financier est positif, mais le ratio de rentabilité du surcoût des semences est à peine acceptable. Pour les sociétés cotonnières, l'impact financier est modeste et est devenu négatif à mesure que le prix mondial du coton est descendu de son record historique, en raison du manque à gagner en quantité de fibre produite, de la réduction en longueur du coton fibre et de l'application d'un malus à la vente à l'exportation. L'image d'un pays fournissant habituellement un coton de qualité est négativement affectée. L'adoption du coton-Bt au Burkina Faso constitue un cas rare d'évolution technologique induisant une divergence d'impacts entre les deux catégories d'acteurs avec une incidence négative potentielle sur le financement de la recherche pour poursuivre le progrès technique.
\end{abstract}

Mots clés : biotechnologie végétale / évaluation d'impact / transformation génétique / variétés / revenu

\begin{abstract}
Financial impacts of Bt-cotton on cotton companies and producers in Burkina Faso. Since 2008, Burkina Faso has been using Bt-cotton through engineered African varieties. The study - covering the 2008-2013 period - is a first ex post assessment of the financial impacts for cotton companies on the one hand, and producers taken on the whole, on the other hand. The study is original in taking into account the imperfect engineering of the varieties used. Impacts were assessed for a real situation of Bt-cotton use compared to a reference situation with no Bt-cotton use. Impacts were also evaluated in comparison to the scenario expected when a decision to adopt was made, for which the assumed $30 \%$ yield increase did not come about. Impacts were evaluated by the partial budget method, considering susceptibility to three criteria in industrial, commercial and world price areas. For farmers, the financial impact was positive, although the profitability rate of the extra cost of Bt cottonseeds was barely acceptable. For cotton companies, impacts were modest and became negative when world price dropped from its historic high as a result of fiber production shortfalls, fiber length decreases and discount at export sales. The commercial image of a country usually supplying quality cotton was negatively affected. The adoption of Bt-cotton in Burkina Faso is a rare case of technological evolution implying diverging impacts to the two main types of players. Potentially, this outcome could hurt the funding mechanism of research for further technical change.
\end{abstract}

Keywords: plant biotechnology / impact assessment / genetic transformation / varieties / income

\section{Introduction}

À partir de 2003, le Burkina Faso a décidé de s'engager dans la voie de l'utilisation du coton génétiquement modifié. Il s'agit plus précisément de variétés rendues résistantes à certains

\footnotetext{
*Auteur de correspondance : michel.fok@cirad.fr
}

ravageurs par l'insertion de gènes tirés de Bacillus thuringiensis (ou coton- $\mathrm{Bt}$ ).

Depuis 2008, l'utilisation du coton-Bt par les paysans est devenue effective. Elle est réalisée avec deux variétés d'origine africaine (FK37 et Stam 59, originaires respectivement du Burkina Faso et du Togo), transformées par Monsanto pour intégrer les deux gènes $\mathrm{Bt}(\mathrm{Cry} 1 \mathrm{Ac}$ et $\mathrm{Cry} 2 \mathrm{Ab})$ à partir du croisement avec une variété américaine porteuse, Coker 312. 
À ce jour, les évaluations des impacts de l'utilisation du coton-Bt au Burkina Faso ont indiqué que les paysans et le pays gagnaient à l'adoption du coton-Bt, mais ce sont des études ex ante. Toutes ces études ont été réalisées par diverses méthodes de modélisation : programmation linéaire (Cabanilla et al., 2005), modèle d'équilibre général appliqué à l'adoption de variétés génétiquement modifiées seulement pour le coton (Elbehri et Macdonald, 2004) ou pour le coton et le maïs simultanément (Vitale et al., 2007).

En dépit du rôle primordial bien connu des sociétés cotonnières dans l'égrenage et la vente de la fibre de coton, elles n'ont pas été prises en compte dans les études citées précédemment. L'accent a été mis seulement sur le cas du fournisseur de la biotechnologie et sur celui des paysans comme utilisateurs de cette technologie.

Pour le Burkina Faso, à l'inverse d'autres pays ayant adopté le coton-Bt, il n'y a pas de réelle évaluation ex post. Les publications reposant essentiellement sur les résultats en milieu contrôlé (Vitale et al., 2008) correspondaient plutôt à des évaluations ex ante, et elles ont contribué à faire retenir, pour les paysans, les hypothèses d'un gain de rendement au champ de $30 \%$ et d'une réduction de quatre traitements insecticides lors de la décision de vulgariser les nouvelles variétés de coton-Bt. Il n'y eut pas d'hypothèse de changement à la transformation industrielle du coton-graine ni à l'exportation du coton fibre, comme cela est habituellement le cas dans tous les pays ayant adopté le coton-Bt.

Les publications à caractère d'étude ex post réalisées au Burkina Faso l'ont été avec un faible recul temporel d'un an ou deux après l'adoption du coton-Bt (Dowd-Uribe, 2014 ; Dowd-Uribe et Bingen, 2011 ; Renaudin et al., 2012). Ces études étaient centrées seulement sur les paysans, à l'opposée des études ex post plus complètes, quoique sans beaucoup de recul dans le temps, aux États-Unis (Zepeda et Nelson, 1997), au Mexique (Traxler et Godoy-Avila, 2004) ou en Afrique du Sud (Gouse et al., 2004).

Le présent article est une première analyse ex post, sur une durée de cinq ans, des impacts financiers à l'échelle nationale de l'utilisation du coton-Bt au Burkina Faso. Il s'appuie sur une étude à partir des données réelles de production pendant la période 2008-2013. Les impacts sont établis et discutés pour les deux acteurs majeurs de cette filière, à savoir les paysans pris globalement et l'ensemble des sociétés cotonnières du pays. Les paysans sont considérés globalement, sans considération de la différenciation en leur sein comme dans les études de Renaudin et al. (2012) et de Dowd-Uribe (2014). La consolidation des impacts pour les deux catégories d'acteurs donne le bilan à l'échelle du pays. À l'inverse des études ex ante citées, les impacts pour la société de biotechnologie impliquée, Monsanto, ne sont pas analysés car les conditions financières de licence d'utilisation des gènes sont entourées de secret.

L'étude réalisée est la première appréhendant les impacts au niveau des sociétés cotonnières. Au Burkina Faso, ce sont les trois sociétés cotonnières (Sofitex, Socoma et Faso-Coton) qui fournissent aux paysans les semences pour la culture et qui leur en achètent le produit - le coton-graine - pour l'égrener et obtenir le coton fibre à vendre, essentiellement à l'exportation. La Sofitex, principale société cotonnière du pays ( $80 \%$ de la production à l'époque), a été l'interlocuteur de Monsanto dans la négociation des conditions d'utilisation des semences de coton-Bt

L'étude a évalué les impacts par la méthode de budget partiel sans cependant l'ambition d'une évaluation parfaitement précise des impacts financiers pour les deux catégories d'acteurs. La méthode évalue les impacts par rapport à une référence définie de situation sans coton-Bt, à partir d'hypothèses jugées plausibles découlant du recoupement $d$ 'information obtenu auprès de divers acteurs de la filière cotonnière. L'étude vise surtout à montrer que les impacts sont divergents entre les deux catégories d'acteurs considérées, positifs pour les paysans mais modestes voire négatifs pour les sociétés cotonnières et que la divergence peut encore durer et s'accentuer. Certains éléments ne sont cependant pas pris en compte dans les budgets partiels réalisés de sorte que la divergence des impacts financiers doit être encore plus marquée que calculée.

L'état des impacts financiers pour les sociétés cotonnières procède de défauts industriel et commercial induits par les variétés de coton-Bt utilisées au Burkina Faso. Ces défauts ont été évoqués par Renaudin et al. (2012) et Dowd-Uribe (2014), mais pas évalués. Ils sont reconnus officiellement à une date récente, d'abord de manière orale ${ }^{1}$ avant que l'information sur le « raccourcissement de la fibre dû à un problème technique » ait été donnée peu après par le directeur de la SOCOMA (AFP, 2015). C'est dans une lettre adressée à Monsanto par une entité du Burkina Faso, pour réclamer des compensations financières liées aux pertes subies par les sociétés cotonnières, que des défauts industriel et commercial sont indiqués ${ }^{2}$. Le contenu de cette lettre infirme ce qui a été clamé auparavant (Airault, 2012).

\section{Matériels et méthodes}

\subsection{Période et données de base}

L'évaluation réalisée couvre la période 2008-2013 (campagnes 2008-09 à 2013-14), c'est-à-dire depuis le début de la vulgarisation du coton-Bt jusqu'à la dernière campagne pour laquelle l'on dispose de données définitives de production.

Les données de base relatives à la production, aux principaux facteurs de production et au marché mondial sont réunies dans le Tableau 1. Elles sont pour l'essentiel disponibles à partir de sources d'accès public, même si elles le sont moins pour les données de production.

Les filières cotonnières des pays de l'Afrique francophone se distinguent par une profusion de données dans divers domaines, notamment dans celui de la production et de l'égrenage industriel, mais ces données sont rarement publiées et diffusées. Le Burkina Faso ne sort pas du lot et se distingue peut-être même par un moindre partage des données comparativement à d'autres pays de la sous-région comme

\footnotetext{
${ }^{1}$ Par un représentant de la Sofitex lors d'une réunion d'un réseau régional sur le coton, les 22 et 23 avril 2015 à Bamako, Mali.

${ }^{2}$ C'est une lettre datée du début de 2015 dont le contenu exact n'est pas connu mais qui évoquerait le problème de raccourcissement du coton fibre et des pénalités subies à la vente à l'exportation pour justifier la demande de dédommagement financier.
} 
Tableau 1. Données de base pour l'estimation des impacts financiers.

Table 1. Basic data for estimating financial impacts.

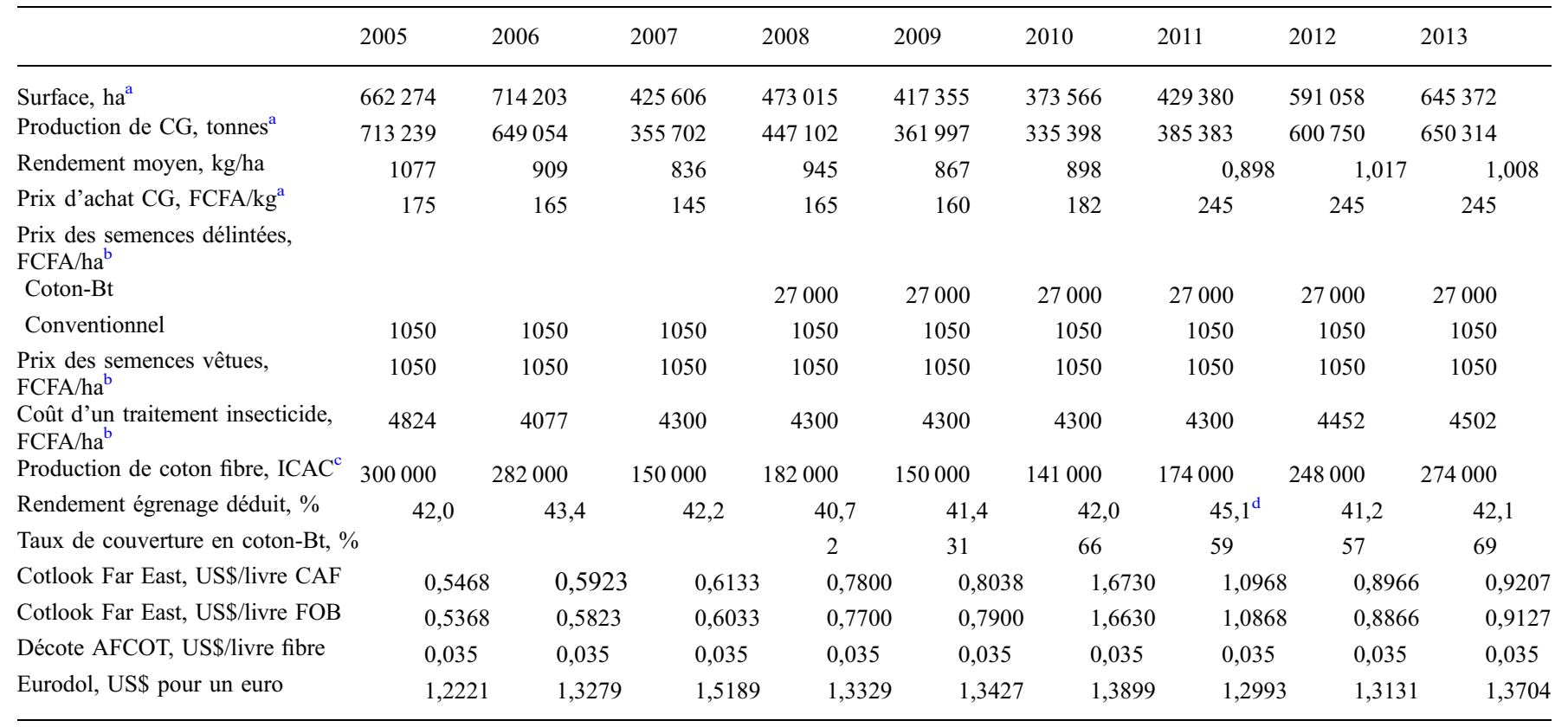

L'année $N$ correspond à la campagne $\mathrm{N} / \mathrm{N}+1 ; \mathrm{CG}$ : coton-graine. Les chiffres de production de coton fibre sont tirés du dernier rapport des statistiques annuelles de l'ICAC de décembre 2015 ; Indice Cotlook en position CAF Extrême Orient pour le type Bola S 1"1/8 du Burkina Faso ; l'indice Cotlook en position FOB est déduit en retenant un différentiel de 0,01 US\$/livre de fibre ; la décote AFCOT est la pénalité appliquée par l'Association française cotonnière pour deux écarts de longueur de la fibre. Le FCFA est à taux fixe de 655,957 pour un euro.

${ }^{\mathrm{a}}$ Informations venant des interlocuteurs des sociétés et de la recherche cotonnière du Burkina Faso s'appuyant notamment sur la note du Ministère de l'Industrie, du Commerce et l'Artisanat établie en 2012 à l'occasion des États Généraux de la filière cotonnière du Burkina Faso.

${ }^{\mathrm{b}}$ Communication des chercheurs du programme coton au Burkina Faso.

${ }^{\mathrm{c}}$ ICAC (2016). Cotton : World statistics.

${ }^{\mathrm{d}} \mathrm{Ce}$ chiffre apparaît anormal et impossible. Il convient de noter que, dans le rapport annuel de l'ICAC de 2012, la production de coton fibre du Burkina Faso de 2011 est indiquée à 151000 tonnes de fibre, pour passer ensuite, dans le rapport de l'année suivante à 174000 tonnes, à surface inchangée, selon les corrections fournies par le Burkina Faso. C'est un ajustement de plus de $15 \%$ jamais constaté pour tout autre pays auparavant. Avec le chiffre initialement communiqué, on aurait eu un rendement égrenage de $39,2 \%$.

la Côte d'Ivoire ${ }^{3}$. Les données utilisées pour la surface et la production de coton-graine sont obtenues par compilation de données communiquées par les interlocuteurs des sociétés et de la recherche cotonnière du Burkina Faso ${ }^{4}$. Les données sont indiquées à partir de l'année 2005 afin de montrer la situation qui prévalait avant la diffusion à grande échelle du coton-Bt.

Les valeurs de production de coton fibre proviennent de l'ICAC, Comité Consultatif International du Coton auquel le Burkina Faso appartient et à qui il doit fournir les statistiques. Ce sont les valeurs de référence les plus officielles. L'on notera

\footnotetext{
${ }^{3}$ La Sofitex est la seule des trois sociétés cotonnières à disposer d'un site Internet où il est possible d'accéder aux statistiques, mais non actualisées, de surfaces cotonnières et de production de coton-graine dans sa zone d'intervention. L'Union nationale des producteurs de coton du Burkina dispose d'un site Internet mais ne fournit aucune information statistique. L'Association interprofessionnelle du coton du Burkina n'a pas de site Internet, contrairement à son homologue en Côte d'Ivoire.

${ }^{4}$ Ils ont communiqué notamment la note du Ministère de l'Industrie, du Commerce et de l'Artisanat préparée pour les États Généraux de la filière cotonnière en 2012 .
}

la valeur déduite de rendement de $45,1 \%$ pour la campagne 2011/12 qui est totalement irréaliste (jamais atteinte en production dans le monde) alors qu'elle avait résulté de la correction d'une statistique antérieurement communiquée et qui donnait un rendement égrenage de 39,2\%. Le niveau de correction est troublant dans la mesure où il sort des cas habituels de correction marginale au niveau de l'ICAC. L'analyse de ces chiffres soutient l'idée que le niveau de rendement égrenage des variétés transformées de coton-Bt devait avoir baissé. Il convient de noter que par calcul ${ }^{5}$, il est possible de déduire le rendement égrenage issu de la production de coton-Bt. Pour un rendement égrenage de 39,2\% de l'ensemble de la production, celui de la production issue du coton-Bt est de $37,7 \%$ si celui du coton conventionnel est de $43 \%$ et si on retient un gain de rendement de $15 \%$ par le coton-Bt couvrant $69 \%$ des surfaces cotonnières.

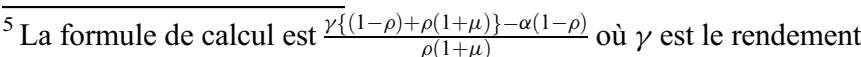
égrenage global, $\alpha$ le rendement égrenage du coton conventionnel, $\rho$ le taux de couverture des surfaces en coton-Bt et $\mu$ le gain de rendement au champ procuré par ce dernier. Avec $\gamma=39,6 \%, \rho=69 \%$, $\mu=15 \%$ et $\alpha=43 \%$, on obtient le résultat de $38,3 \%$ pour le rendement égrenage du coton-Bt.
} 
La référence de prix mondial pour l'exportation du coton des pays de l'Afrique francophone est l'indice Cotlook pour les ventes en position CAF en Extrême-Orient. Les ventes à l'exportation du Burkina Faso étant réalisées en position FOB, l'indice est ajusté en tenant compte d'un différentiel de 1 cent américain par livre de coton fibre mais la qualité de précision sur la valeur de ce différentiel a peu d'incidence sur les bilans réalisés.

Pour le taux de change entre le dollar américain et l'euro, les données des séries de la Banque de France sont utilisées pour établir une moyenne annuelle de décembre de l'année $N$ à juin de l'année $N+1$ afin de correspondre à la période d'embarquement du coton exporté. La conversion en FCFA se fait suivant le taux fixe de 655,957 FCFA pour un euro.

\subsection{Situation de référence pour évaluer les impacts}

La situation de référence pour évaluer les impacts de l'adoption du coton-Bt est à établir car, dans une approche par budget partiel, les impacts financiers sont estimés à partir des variations de coûts et de recettes pour les paysans et les sociétés cotonnières par rapport à cette situation de référence.

La situation de référence correspond à la continuation de l'utilisation exclusive de variétés de coton conventionnel, sur des superficies telles qu'elles ont été réalisées comme indiquées dans le Tableau 1, avec des variétés de coton conventionnel ayant donné habituellement $43 \%$ de rendement à l'égrenage et une fibre de longueur 1 "1/8 en quasi-totalité ${ }^{6}$.

Les rendements au champ des deux types de coton, données manquantes pour les calculs des recettes des paysans et sociétés cotonnières, peuvent être déduits ${ }^{7}$ des productions et surfaces réellement réalisées pendant la période 2008-2013.

L'établissement de la situation de référence requiert de connaître la part des superficies en coton-Bt et le gain de rendement procuré par ce dernier. Les informations obtenues sont reproduites dans le Tableau 1.

L'augmentation réelle du rendement au champ a été inférieure à celle qui a été clamée ou espérée. Renaudin et al. (2012) n'ont pas observé de gain de rendement découlant du coton-Bt, observation confirmée dans la zone de la Socoma (Sigrist, 2014). Dowd-Uribe (2014) a par contre rapporté que le coton-Bt avait induit un gain de rendement, sans cependant en préciser le niveau, mais en indiquant que seulement les paysans mieux équipés en avaient profité. La variation du gain issu du coton-Bt selon les paysans ressort aussi dans l'interview du directeur de la Sofitex (Tao, 2012). Les calculs sont effectués avec l'hypothèse d'un gain de rendement de $15 \%$ en moyenne sur l'ensemble de la période étudiée, selon les informations obtenues des chercheurs du programme coton du Burkina Faso (moitié moindre que les $30 \%$ espérés) sans disposer cependant des valeurs réelles des différences de rendement pour chaque année.

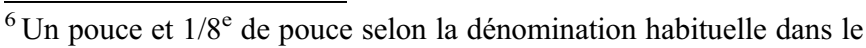
négoce du coton. En réalité, le Burkina Faso produisait essentiellement cette longueur de fibre, avec une proportion assez substantielle audessus de cette longueur.

${ }^{7}$ Le rendement du coton conventionnel est $r_{1}=\frac{P}{S} \times 1 /(1+\rho \mu)$ et celui du coton-Bt est $(1+\mu) r_{1}$ où $P$ et $S$ sont la production totale de coton-graine et la surface totale en coton, $\rho$ le taux de couverture des surfaces en coton-Bt et $\mu$ le gain de rendement procuré par ce dernier.
}

\section{3 Éléments de calcul pour le bilan financier des paysans producteurs}

L'étude concerne les bilans financiers et non économiques, aussi ne sont pas valorisés les autres avantages (ou inconvénients) que les paysans peuvent avoir ressenti en utilisant le coton-Bt. Les variations en termes de quantité ou de pénibilité de travail ne sont donc pas valorisées.

Par la méthode de budget partiel, les bilans financiers des paysans et des sociétés cotonnières découlent des gains et pertes pour les postes de recettes ou de dépenses affectés par l'utilisation du coton-Bt. La méthode est préférée à celle du surplus économique qui est plus difficile d'application en méconnaissance des fonctions de production, surtout dans le cas des sociétés cotonnières.

Pour les paysans, les gains financiers sont issus d'une part du surcroît de production de coton-graine par l'augmentation de $15 \%$ du rendement et d'autre part de l'économie de quatre traitements insecticides. Ces gains sont contrebalancés par le surcoût en semences pour utiliser le coton-Bt. L'hypothèse de l'économie de quatre traitements insecticides à l'échelle nationale est plausible car cette économie était le message technique essentiel à la vulgarisation même s'il n'était pas ou mal parvenu à tous les paysans dans les premières années d'utilisation du coton-Bt (Renaudin et al., 2012).

Le surcoût dans l'utilisation des semences de coton-Bt est estimé dans un contexte où la production de semences délintées ne permettait pas de couvrir toutes les superficies à emblaver. Il est retenu que le coton-Bt était cultivé avec des semences délintées ou vêtues (sans ou avec le duvet recouvrant les graines) aux doses respectives de 15 et $30 \mathrm{~kg} /$ ha suivant les prix de cession indiqués dans le Tableau 1. En absence d'information divulguée sur la distribution des deux types de semences, le surcoût des semences a été calculé pour les surfaces en coton-Bt, en considérant qu'elles ont été couvertes aux taux de $2 / 3-1 / 3$ respectivement en semences délintées et vêtues.

Les autres variations de coûts de production au niveau des paysans ne sont pas prises en compte, de sorte que leur bilan financier global est surestimé. L'utilisation de semences de coton- $\mathrm{Bt}$, en raison d'un potentiel de rendement meilleur, peut amener les producteurs du petit paysannat à modifier l'intensification dans l'utilisation des autres intrants en fonction de leur degré de contrainte en ressources financières. Les plus nantis tendent à intensifier davantage, à l'inverse des plus dépourvus (Qayum et Sakkhari, 2005), c'est une variation d'intensification qui est conforme à la différenciation des paysans soulignée par Renaudin et al. (2012) et Dowd-Uribe (2014). Globalement, pour l'ensemble des paysans, l'effet peut s'annuler. Par contre, une augmentation de rendement induit forcément un surcroît en travail à la récolte, parfois avec embauche de main d'œuvre rémunérée qui n'est pas prise en compte ici.

Pour cerner la rentabilité pour les paysans d'adopter le coton-Bt, dont l'utilisation induit un coût marginal substantiel issu de la forte augmentation du prix des semences, le ratio bénéfice/coût est calculé par le rapport de la valeur du supplément de production sur le surcoût en intrants (coût des semences diminué par l'économie en insecticide). Ce ratio est à rapprocher du taux de risque financier des intrants - rapport de la valeur de la production sur le coût total des intrants monétaires, soit l'inverse du ratio retenu ici - que les sociétés 
cotonnières en Afrique francophone avaient le souci de maintenir à moins de $30 \%$ (ou ratio de 3 ) jusqu'au début des années 1990 (Fichet, 1996).

\section{4 Éléments de calcul du bilan financier des sociétés cotonnières}

Dans l'analyse par budget partiel par rapport à la situation de référence, tous les coûts et gains supplémentaires pour les sociétés cotonnières ne sont pas considérés.

Le seul coût supplémentaire considéré avant l'égrenage et la commercialisation est le supplément de paiement du surcroît de production des paysans. C'est ce paiement qui fait la recette principale des paysans analysée plus haut. Les autres coûts liés à la commercialisation, au transport et à l'égrenage du supplément de production ne sont pas considérés faute d'information. Il en découle une imprécision dans les impacts financiers des sociétés cotonnières, impacts qui sont donc surestimés s'ils sont positifs et sous-estimés s'ils sont négatifs.

Les gains découlent des recettes du supplément de coton fibre venant de l'égrenage du supplément de production de coton-graine des paysans. Ces gains sont calculés à partir du surplus de quantité de fibre valorisés au prix mondial pour la qualité produite.

Le surplus en quantité de fibre est le bilan des productions issues respectivement du coton-Bt et du coton conventionnel, chacune étant déduite de l'application du rendement égrenage à la production de coton-graine.

La valeur à l'exportation du coton fibre est calculée à partir des niveaux du prix mondial indiqués dans le Tableau 1, à pondérer s'il y a perte de qualité de la fibre produite. Dans cette manière de calculer, on suppose implicitement que les deux types de production issus des deux types de coton sont égrenés et commercialisés séparément.

\subsection{Scénario anticipé non avéré}

À titre de rappel, le scénario anticipé par les acteurs de la filière cotonnière du Burkina Faso pour adopter le coton-Bt reposait sur un gain de rendement au champ de $30 \%$, au prix d'un surcoût des semences partiellement compensé par une économie de quatre traitements insecticide et sans changement industriel à l'égrenage ni de changement commercial à l'exportation.

Les éléments fournis ci-dessus permettent de constater que le scénario anticipé ne s'est pas concrétisé. Les impacts financiers de ce scénario ont cependant été calculés pour voir à quoi ils auraient pu correspondre.

\section{6 "Scénario plausible » de pertes industrielle et commerciale}

Les acteurs de la filière cotonnière au Burkina Faso avaient refusé d'utiliser les variétés proposées par Monsanto et avaient exigé la transformation de deux variétés africaines qu'ils ont fournies. Une telle exigence procède du souci d'utiliser des variétés adaptées au contexte local.

La qualité de la transformation des deux variétés africaines fournies par la filière coton du Burkina Faso a cependant été imparfaite et ne pouvait que l'être, compte tenu du faible délai de réalisation. Selon les informations recoupées auprès des chercheurs du programme coton du Burkina Faso, les variétés africaines ont été fournies pour transformation entre 2003 et 2006 et les semences transformées ont été renvoyées pour utilisation en 2007 après seulement deux rétrocroisements. Les variétés de coton-Bt utilisées ne pouvaient alors correspondre aux attentes des acteurs du Burkina Faso qui voulaient que les variétés transformées gardent totalement les qualités des variétés d'origine ${ }^{8}$. Il ne s'agit d'ailleurs pas seulement d'une question de nombre insuffisant de rétrocroisements. En effet, si les travaux menés en Australie (Stiller et al., 2006) indiquent que trois rétrocroisements peuvent suffire pour transformer les variétés en gardant les caractéristiques désirées du parent local, c'est seulement sous la condition de la mise en œuvre d'une sélection efficace sur les critères agronomiques et qualitatifs de la fibre. Comme les gènes commandant ces caractéristiques ne sont pas liés aux gènes $\mathrm{Bt}$, la sélection doit même être entretenue après la commercialisation des variétés obtenues.

L'inachèvement de la transformation des deux variétés africaines a conduit à garder dans les variétés transformées utilisées les traits du parent américain employé dans la transformation. Sur le plan de la qualité de la fibre, ces traits sont très éloignés de ceux des variétés africaines et constituent de fait des défauts sur le marché mondial. Le parent américain Coker 312 est une variété datant de 1971 et est typique des variétés américaines. Il donne une fibre courte, au plus de 1"1/16 selon l'analyse de Kuraparthy et Bowman (2013) sur l'évolution des variétés américaines en matière de longueur de la fibre. C'est au moins deux grades de longueur, libellés en $1 / 32^{\mathrm{e}}$ de pouce, de moins que la fibre des variétés en Afrique francophone. Par ailleurs, le rendement égrenage est un caractère jamais sélectionné aux États-Unis, contrairement en Afrique francophone qui bénéficie ainsi du niveau le plus élevé dans le monde. Aussi la variété Coker 312 était inférieure en rendement égrenage aux variétés africaines.

L'évaluation des impacts financiers pour les sociétés cotonnières est réalisée pour le scénario reposant sur l'hypothèse d'une perte industrielle découlant d'un rendement égrenage moindre du coton-Bt, au niveau de $40 \%$ contre $43 \%$ pour le coton conventionnel.

Sur le plan commercial, les hypothèses reposent sur l'application d'une décote sur la fibre plus courte issue du coton-Bt et sur l'imposition d'un malus à la vente à l'exportation de tout le coton produit à partir de $2010^{9}$. Les calculs sont réalisés selon l'hypothèse d'un malus de $10 \mathrm{FCFA} / \mathrm{kg}$ de fibre.

La décote à la fibre issue du coton-Bt - en référence au prix mondial pour un niveau de longueur de la fibre de coton (Fok, 2004), 1"1/8 dans le cas présent - équivaut à celle de deux écarts de longueur suivant la grille des écarts de l'Association française cotonnière, dépositaire du système

\footnotetext{
${ }^{8} \mathrm{Au}$ regard du taux de pureté variétale de $99 \%$ (certains pays vont audelà, comme l'Espagne), il convient de noter qu'après deux rétrocroisements, on retrouve seulement $87,5 \%$ du génotype de la variété d'origine contre $98,4 \%$ au bout de cinq rétrocroisements.

${ }^{9}$ En considérant un délai de deux campagnes pour que les négociants se rendent compte de la dépréciation de la qualité du coton burkinabé, comparativement à ce qui prévalait avant l'adoption du coton-Bt et relativement au coton des autres pays de la sous-région.
} 
de règlements européen par lequel le coton burkinabé est vendu. L'hypothèse sur l'application d'un malus à la vente à l'exportation peut procéder d'une longueur de fibre devenue moins uniforme ${ }^{10}$ (caractère fort important aux yeux des filateurs) qui peut amener les négociants à appliquer le malus retenu dans les calculs.

\subsection{Vérification de la validité des résultats de pertes industrielle et commerciale}

Pour vérifier la validité du résultat de pertes financières pour les sociétés cotonnières selon les hypothèses retenues, les calculs de sensibilité sont réalisés pour différents niveaux du rendement égrenage des variétés transformées de coton-Bt et différents niveaux du prix mondial ainsi que pour une valeur plus élevée de la décote pour fibre courte $(5,0 \mathrm{vs}$. 3,5 cents US/ livre). Les résultats ont été faits pour la campagne 2013-14. Le choix de cette campagne est justifié car elle correspond à un régime « de croisière » avec un taux de couverture des surfaces en coton-Bt de $60 \%$. Elle a été marquée aussi par un niveau du prix mondial plus proche, quoique toujours supérieur, au prix de 0,80 US\$/livre dégagé des analyses à long terme de l'ICAC.

La sensibilité des résultats au taux de rendement égrenage est conduite parce que le taux retenu de $40 \%$ pour les variétés transformées est approximatif selon les informations obtenues. Le taux de rendement égrenage peut avoir été différent entre les deux variétés transformées et leurs emblavements peuvent avoir été ajustés en défaveur de la variété à rendement égrenage moindre. C'est aussi un défaut qui peut être corrigé, au moins partiellement, par une adaptation du procès industriel à l'égrenage, en réduisant la vitesse de rotations des scies, d'une part et d'autre part, en réduisant le niveau de nettoyage de la fibre après égrenage. Ce sont des adaptations qui ont néanmoins un coût supporté par les sociétés cotonnières. Elles peuvent aboutir à une fibre plus chargée et qui peut donner aux négociants un autre prétexte pour appliquer le malus.

L'hypothèse d'une décote plus élevée - pour raccourcissement de la fibre - a été considérée car le décrochement du segment de qualité (plus précisément le segment de longueur de la fibre) occupé habituellement par le Burkina Faso, et que les autres pays de l'Afrique francophone continuent à occuper, place le pays concerné en position de faiblesse dans la négociation du prix de vente à l'exportation.

La sensibilité des résultats au prix mondial a également été effectuée parce que la période d'adoption du coton-Bt au Burkina Faso a coïncidé avec la période d'une très grande volatilité du prix mondial et surtout quelques années de prix mondial bien supérieur à la tendance historique de ce prix.

\section{Résultats et discussion}

Le Tableau 2 donne les résultats des calculs des impacts pour les deux scénarios par rapport à la situation de

\footnotetext{
${ }^{10}$ Un tel résultat peut découler automatiquement de l'égrenage de production à fibre de longueur différente.
}

référence sans coton-Bt : « scénario plausible » avec pertes industrielle et commerciale, d'une part et « scénario anticipé » non avéré, d'autre part. Une présentation plus complète avec indication des formules de calculs pour le «scénario plausible » est reproduite au Tableau 3.

\subsection{Une adoption sans explosion de la production}

Au cours de la période 2008-2013, l'évolution de la superficie et de la production cotonnière ne témoigne pas d'effet, en tout cas pas d'effet immédiat de l'adoption du cotonBt (Fig. 1). L'adoption du coton-Bt n'a pas induit une impulsion immédiate à la production. La baisse de la production depuis 2005 s'est poursuivie après l'adoption du coton-Bt.

Les superficies ont ainsi répondu au prix d'achat du coton-graine, même si bien d'autres facteurs interviennent, comme ceux liés au fonctionnement de la filière cotonnière (Fok, 2010 ; Kaminski et al., 2011). Lorsque le prix d'achat a dépassé le niveau prévalant en 2005 , les superficies ont été proches du record d'emblavement de 2006/7. Cette évolution a bénéficié de la forte montée du prix mondial à partir de 2010 (et qui n'a commencé à se résorber que depuis mi2014). Cette évolution des superficies, peu ou pas dépendante de l'adoption du coton-Bt, justifie la validité de considérer les mêmes superficies dans le scénario de référence.

La production de coton-graine a suivi la même évolution que pour les superficies, sans dépasser le record de production de 2005. Les courbes des superficies et des productions tendent à se confondre, indiquant une augmentation pour atteindre un rendement moyen au champ d'une tonne à l'hectare. L'adoption du coton-Bt a contribué à cette augmentation récente du rendement, car les conditions climatiques en 2012 et 2013 n'ont pas été particulièrement favorables, et cela concourt à confirmer l'hypothèse de gain de rendement retenue dans le scénario plausible alors que Renaudin et al. (2012) n'en avaient pas observé lors de la campagne 2009-10 dans la seule mais principale zone de production de la Sofitex.

\subsection{Impacts financiers pour les paysans : positifs mais à nuancer}

Au regard du « scénario plausible », le gain de revenu de l'ensemble des paysans a progressé tout au long de la période considérée. Les recettes de la commercialisation du surplus de production de coton-graine ont dépassé largement le surcoût en intrants induit par l'utilisation du coton-Bt.

Le gain de revenu observé procède du gain de rendement au champ de $15 \%$, mais sa progression a surtout dépendu de l'augmentation des surfaces et du prix d'achat du cotongraine, à la faveur de la montée du prix mondial du coton. Cela avait été souligné dans le cas de l'Afrique du Sud (Gouse et al., 2004).

L'utilisation du coton-Bt a induit un investissement financier supplémentaire dont le ratio bénéfice/coût reste cependant modeste en situation normale de marché mondial. Ce ratio est de l'ordre de deux en dehors d'épisode de flambée anormale du prix mondial. C'est le niveau minimum 
Tableau 2. Impacts financiers du « scénario plausible » au regard de la situation de référence et du scénario anticipé à la décision d'adopter le coton-Bt.

Table 2. Financial impacts of the plausible scenario with regard to the situation of reference and to the scenario anticipated while deciding adopting Bt-cotton.

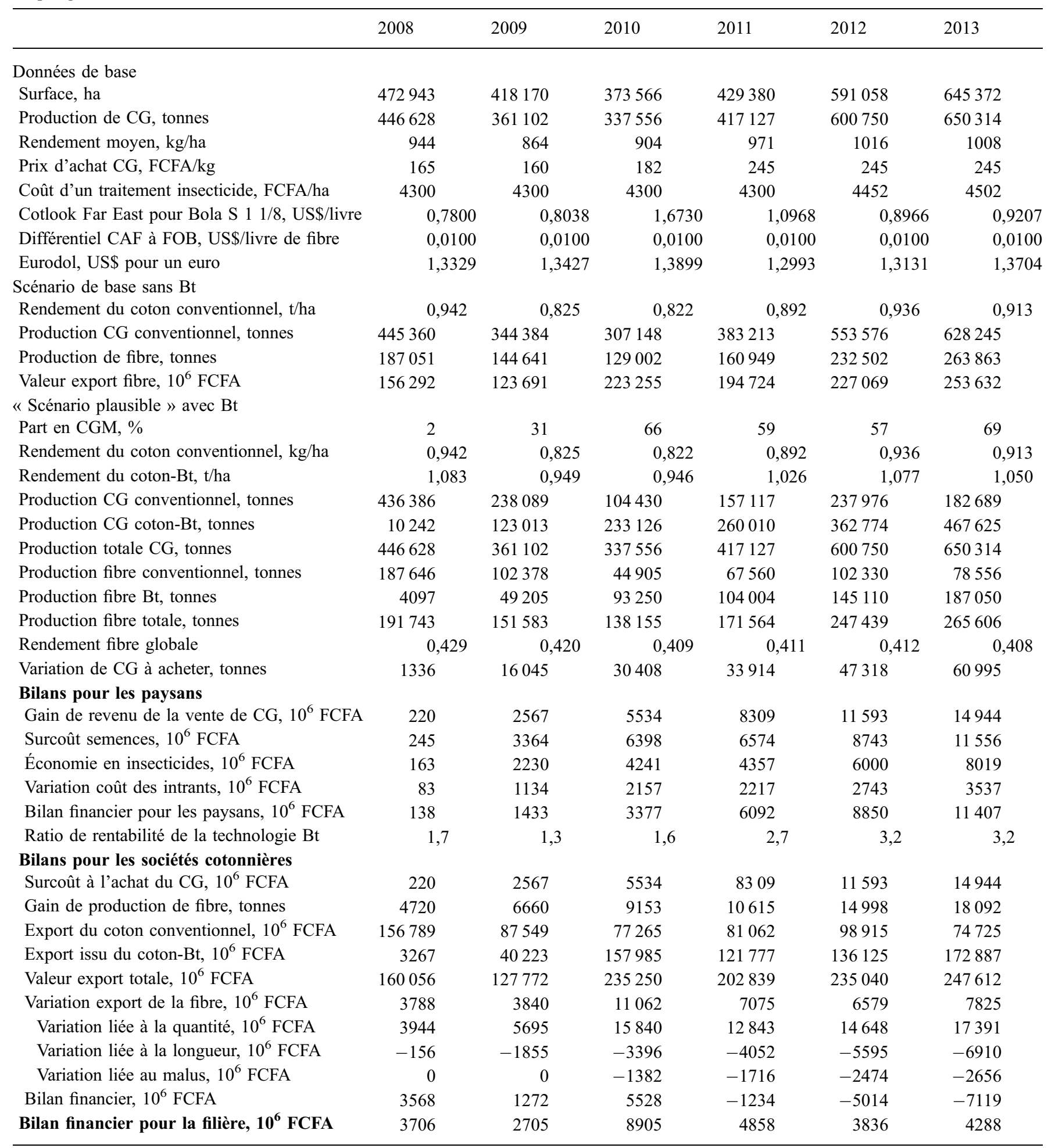

CG : coton-graine ; variation ou gain : différence avec le scénario de référence sans coton-Bt. 
Tableau 3. Détail des calculs des impacts financiers du scénario plausible.

Table 3. Calculation details of the financial impacts for the plausible scenario.

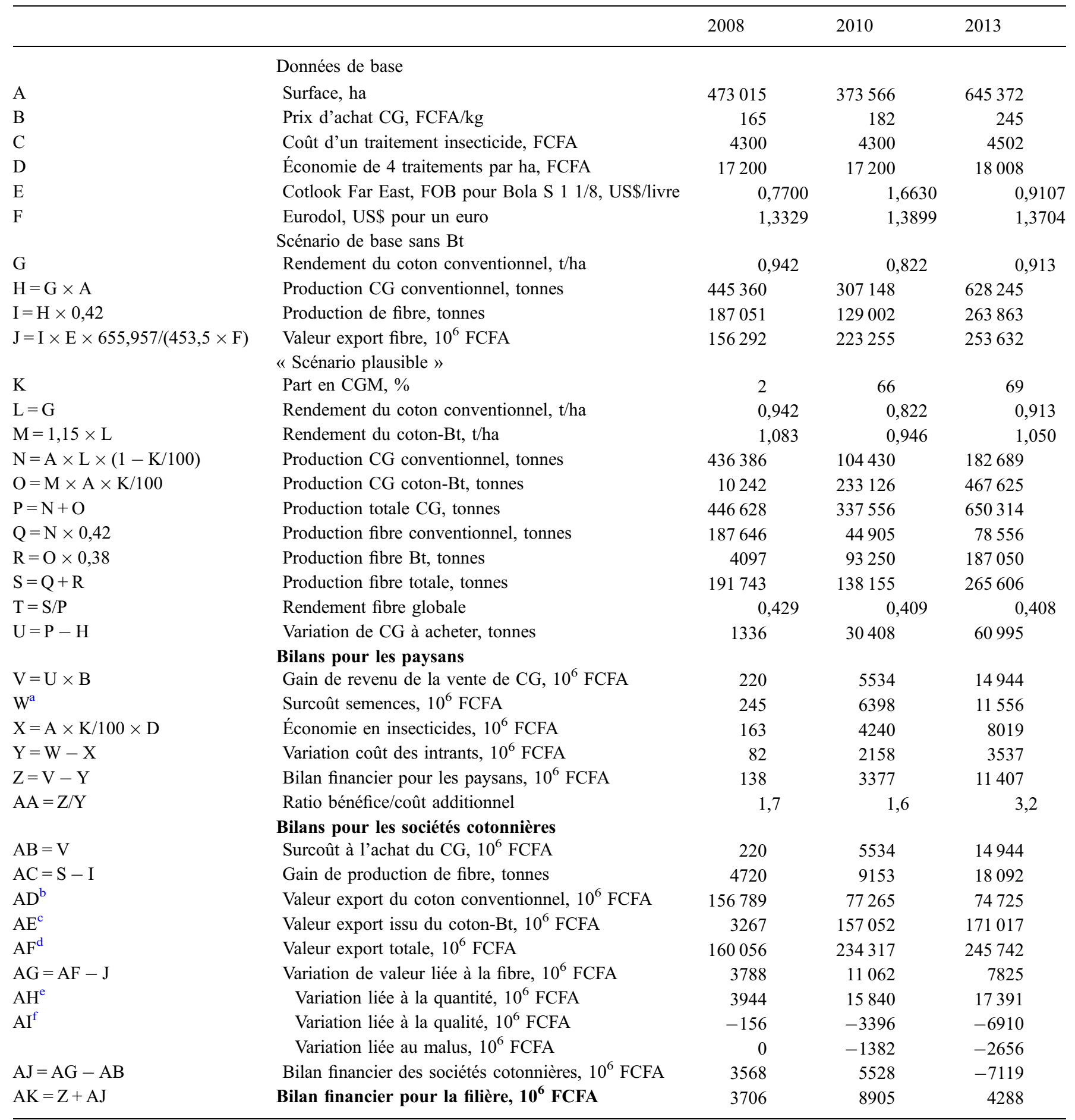

Une livre $=0,4535 \mathrm{~kg}$; Décote de qualité : 0,035 par livre.

${ }^{a}$ Calculé selon les prix des semences vêtues et délintées indiquées dans le Tableau 1.

${ }^{\mathrm{b}} \mathrm{AD}=\mathrm{Q} \times \mathrm{E} / 0,4535 / \mathrm{F} \times 655,957 / 1000$ avec déduction d'un malus de $\mathrm{S} \times 10 \times 1000$ à partir de 2010.

${ }^{\mathrm{c}} \mathrm{AE}=\mathrm{R} \times(\mathrm{E}-0,035) / 0,4535 / \mathrm{F} \times 655,957 / 1000$ avec déduction d'un malus de $\mathrm{S} \times 10 \times 1000$ à partir de 2010.

${ }^{\mathrm{d}} \mathrm{AF}=\mathrm{AD}+\mathrm{AE}$.

${ }^{\mathrm{e}} \mathrm{AC} \times \mathrm{E} / 0,4535 / \mathrm{F} \times 655,957 / 1000$.

${ }^{\mathrm{f}} \mathrm{R} \times 0,035 / 0,4535 / 1,3329 \times 655,957 / 1000$. 


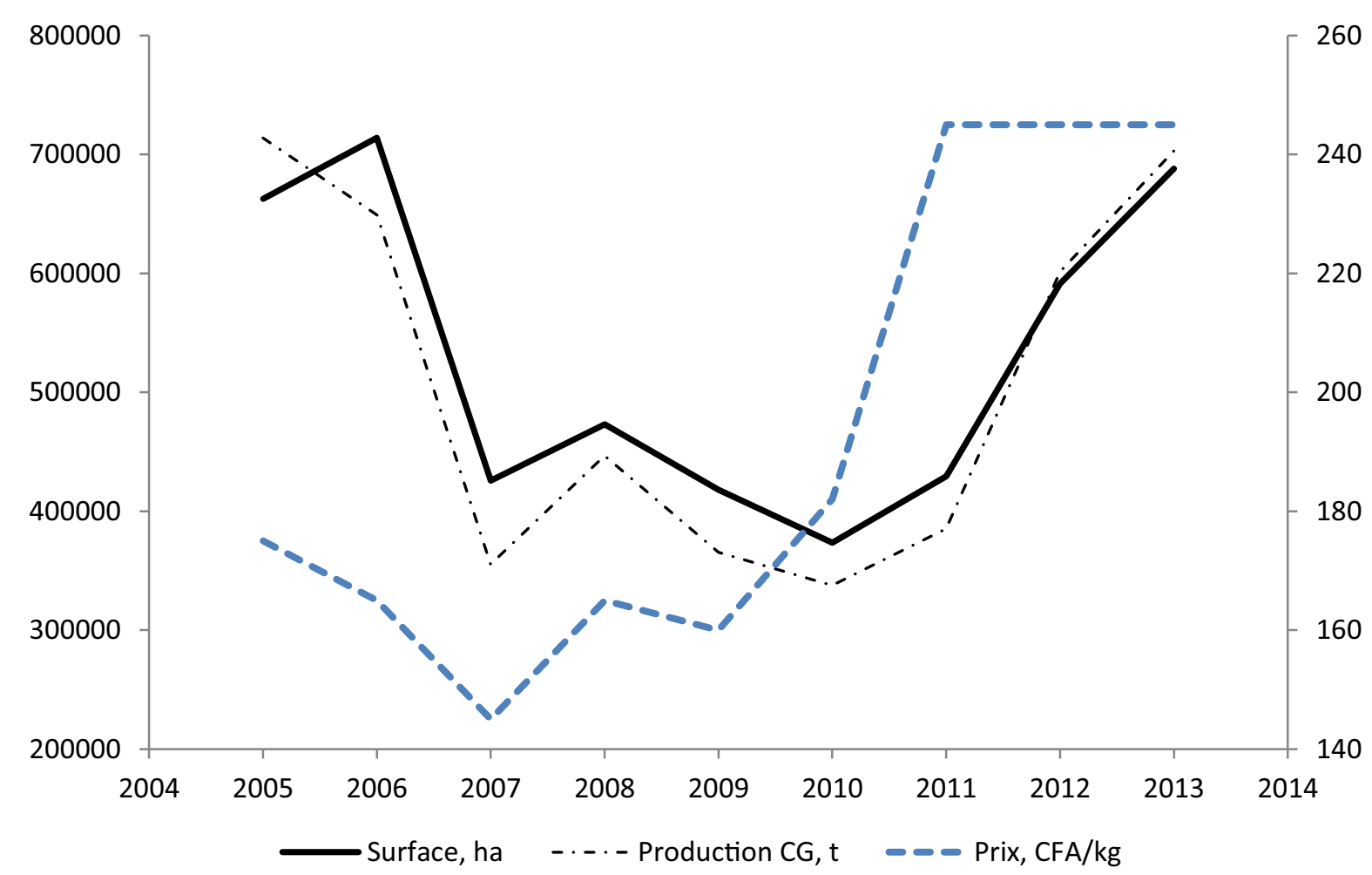

Fig. 1. Évolution des surfaces et productions cotonnières en relation avec le prix d'achat aux producteurs.

Fig. 1. Evolution of cotton areas and productions related to the purchase price to producers.

habituellement considéré pour espérer l'adhésion des paysans à une nouvelle technologie. Il équivaut à un coefficient de risque financier de $50 \%$, contre les $30 \%$ habituellement retenus.

Sur l'ensemble de la période étudiée, les gains cumulés des paysans ont été de 31,3 milliards de FCFA. C'est cependant moins de la moitié des 74,1 milliards si le gain anticipé de rendement de $30 \%$ avait été obtenu (résultats non reproduits).

\subsection{Des gains modestes aux pertes financières pour les sociétés cotonnières}

Suivant le « scénario plausible » retenu, la production de fibre a augmenté, de manière irrégulière sous les effets des variations de surface et du moindre rendement égrenage du coton-graine issu des semences de coton-Bt.

La variation de la valeur à l'exportation du coton fibre - par rapport au scénario de référence - a augmenté, mais elle n'a pas été positivement influencée par les années de prix mondial élevé à causes des coûts commerciaux liés à la fibre plus courte et à l'application d'un malus. Ainsi, les pénalisations commerciales ont représenté plus de la moitié de la valeur du surplus de fibre à exporter.

Pour toute la période étudiée, le bilan financier des sociétés cotonnières, une fois tenu compte du paiement du surplus de coton-graine aux paysans, est devenu négatif durant les trois dernières années de la période d'étude, au cours desquelles le prix mondial restait encore très favorable. C'est la conséquence de l'application d'un malus sur toute la production mais aussi du prix d'achat aux paysans qui a été ajusté par rapport au prix mondial.
Par rapport au " scénario anticipé » mais non avéré, les impacts financiers sont totalement opposés à ce qui était attendu (résultats non reproduits). La situation est déficitaire de 3,0 milliards sur l'ensemble de la période, au lieu d'un gain cumulé de 124,0 milliards, soit un différentiel de 127,0 milliards FCFA.

\subsection{Un bilan global modeste pour la filière}

Globalement, le bilan consolidé des paysans et des sociétés cotonnières a été positif chaque année pendant la période étudiée, mais de manière modeste même quand le prix mondial est resté élevé les trois dernières années de la période. C'est le résultat des faibles gains voire des pertes subies par les sociétés cotonnières. C'est une situation inattendue, paradoxale voire ironique, car ce sont ces dernières, sous la houlette de la Sofitex, principale société cotonnière, qui ont promu l'utilisation du coton-Bt dans l'espoir d'un gain de compétitivité.

Par rapport au « scénario anticipé » mais non avéré, le bilan global cumulé sur la période étudiée a été de 28,3 milliards, au lieu d'un gain cumulé de 198,2 milliards FCFA.

\subsection{Un cas de changement technologique à impacts financiers divergents}

La divergence des bilans financiers entre les deux catégories d'acteurs - systématiquement positifs pour les paysans et plus fréquemment négatifs pour les sociétés cotonnières - est un résultat qui n'était pas du tout anticipé lors de l'adoption du coton-Bt. Il convient de souligner que la divergence des impacts 
à l'intérieur de chaque catégorie d'acteurs n'est pas appréhendée dans l'étude car les deux catégories d'acteurs sont considérées globalement. L'existence de la divergence au sein des paysans dans les premières années de l'utilisation du coton-Bt a été signalée (Dowd-Uribe, 2014 ; Renaudin et al., 2012), notre étude ne permet pas de confirmer ou de chiffrer cette divergence sur une période plus longue. Il n'y a pas travaux abordant la différence des impacts entre les trois sociétés cotonnières, mais l'on peut supputer son existence du fait de leurs différences dans les infrastructures industrielles (d'ancienneté diverse) et dans l'expérience cotonnière.

La divergence des bilans des deux catégories d'acteurs est une situation rarement rencontrée dans les filières cotonnières des pays de l'Afrique francophone. La divergence des bilans révèle que l'adoption du coton- $\mathrm{Bt}$, dans les conditions que le Burkina Faso a connues, n'a pas été Pareto-efficace.

Le résultat paradoxal pour les sociétés cotonnières établit une situation nouvelle en matière de changement technologique. Avant le milieu des années 1980, l'augmentation du rendement au champ sans augmentation du rendement égrenage faisait gagner les paysans mais aussi les sociétés cotonnières. Par la suite, l'augmentation du rendement égrenage sans augmentation notable du rendement au champ faisait gagner les sociétés cotonnières mais sans faire perdre les paysans. Avec l'adoption des variétés mal transformées de coton- $\mathrm{Bt}$, les sociétés cotonnières perdent souvent et de manière substantielle, alors que la filière prise globalement gagne peu.

La divergence observée des impacts entre les deux catégories d'acteurs, si elle n'est pas résolue, pose la question de l'avenir $\mathrm{du}$ progrès technique dans la filière coton du Burkina Faso. N'ayant pas obtenu les compensations réclamées à Monsanto, les sociétés cotonnières ont souhaité réduire fortement voire annuler les surfaces couvertes en coton-Bt, proposition qui n'a pas reçu la pleine adhésion des représentants des paysans. La divergence d'impacts peut se traduire par une désolidarisation entre les deux catégories d'acteurs et nuire à leur consensus dans l'orientation de la recherche pour gagner en productivité et compétitivité dans le futur.

\subsection{Divergence des impacts financiers sous-estimée par la méthode d'estimation}

Même si la divergence des impacts financiers entre les deux catégories d'acteurs n'est pas remise en cause, le degré de divergence est affecté par l'application imparfaite de la méthode des budgets partiels. Tous les éléments à prendre en compte dans les budgets partiels ne l'ont pas été de manière complète et parfaite. Pour les paysans, le bilan financier est positif mais il est surestimé car l'augmentation des coûts de production - pour faire face à une production plus élevée dans une agriculture peu mécanisée et à économies d'échelle faibles voire nulles - n'a pas été prise en compte. Pour les sociétés cotonnières, d'un côté le caractère négatif du bilan financier est surestimé car n'a pas été prise en compte la réduction des coûts de la commercialisation du coton-graine jusqu'à l'égrenage en conséquence d'un plus grand niveau d'utilisation des capacités installées. De l'autre, ce degré négatif est sous-estimé par la non-intégration des pénalités liées aux réclamations des clients mécontents du coton livré. Il se peut que l'élément sous-estimé ait été plus important que l'élément surestimé.
Une autre raison indiquant que le bilan financier des sociétés cotonnières a été sous-estimé provient de la méthodologie suivie pour distinguer dans les calculs, les productions issues respectivement du coton- $\mathrm{Bt}$ et du coton conventionnel. La distinction est justifiée si les deux productions sont égrenées séparément et si les balles de coton constituent des lots séparés et qui sont vendus séparément. Il est probable qu'il n'en soit pas du tout ainsi dans la réalité car il n'est pas indiqué que les deux types de coton sont distingués depuis la commercialisation au niveau des villages. Il en découle que la décote pour fibre courte puisse être appliquée sur une part plus importante de la production que ce qui a été considéré.

Enfin, les pertes financières des sociétés cotonnières sont incomplètement estimées avec la seule prise en compte du malus imposé par les négociants. Le Burkina Faso jouissait d'un bonus, le différentiel total est la somme du bonus perdu et du malus nouvellement imposé. Les pertes liées à l'effacement du bonus ne sont pas intégrées dans les calculs faute de la connaissance du niveau du bonus antérieurement appliqué.

\subsection{Sensibilité du bilan financier des sociétés cotonnières aux paramètres industriel, commercial et du marché mondial}

Les résultats des analyses de sensibilité indiquent que ni le caractère négatif du bilan financier ni l'importance du bilan négatif n'ont été induits par les hypothèses retenues dans les calculs et qui leur auraient été défavorables (Fig. 2).

Le bilan financier des sociétés cotonnières est sensible au niveau du rendement égrenage, à l'inverse de celui des paysans qui y est totalement insensible (Fig. 2, Box a et Box b). Le niveau de décote induite par la perte de longueur du coton fibre n'a pas d'incidence sur la divergence des bilans financiers entre les sociétés cotonnières et les paysans. Ces résultats sont la conséquence directe du mode de rémunération des paysans : ils sont payés à la commercialisation du coton-graine et ils ne sont pas concernés directement ${ }^{11}$ par la vente du coton fibre.

Avec une décote de 3,5 cents US/livre, il faut un rendement égrenage du coton-Bt proche de $42 \%$ (comparativement à $43 \%$ pour le coton conventionnel) pour que le bilan financier des sociétés cotonnières ne soit plus négatif (Fig. 2, Box a). Avec une valeur de la décote plus élevée, le bilan reste négatif même si le rendement égrenage du coton-Bt parvient à atteindre $42 \%$ (Fig. 2, Box b).

À l'opposé, si le rendement égrenage du coton-Bt est inférieur à $40 \%$, le caractère négatif du bilan peut se creuser de beaucoup, encore plus à une valeur plus élevée de la décote.

Le caractère négatif du bilan financier des sociétés cotonnières observé dans les dernières années de la période étudiée a même été pondéré par le prix mondial du coton fibre prévalant pendant la période étudiée et dont le niveau a été bien supérieur à celui correspondant à la tendance historique de ce prix. Cela est encore plus vrai avec une valeur plus

\footnotetext{
${ }^{11}$ Selon le mécanisme de stabilisation du prix du coton-graine payé aux paysans, « mécanisme de lissage », ces derniers sont peu ou prou concernés par le sur-bénéfice éventuel à l'exportation du coton fibre. Mais la redistribution aux paysans n'est effectuée que sous la condition d'un abondement suffisant du fonds de lissage.
} 
Box a : Variation du bilan financier des sociétés cotonnières pour une décote de 3,5 cents US/livre de la fibre exportée et selon le rendement égrenage des variétés Bt transformées pour la production de 2013

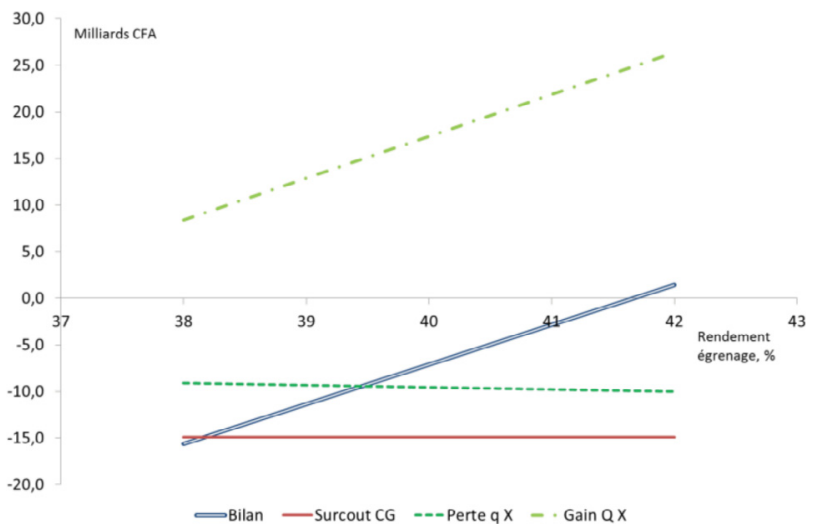

Box b : Variation du bilan financier des sociétés cotonnières pour une décote de 5,0 cents US/livre de la fibre exportée et selon le rendement égrenage des variétés Bt transformées pour la production de 2013

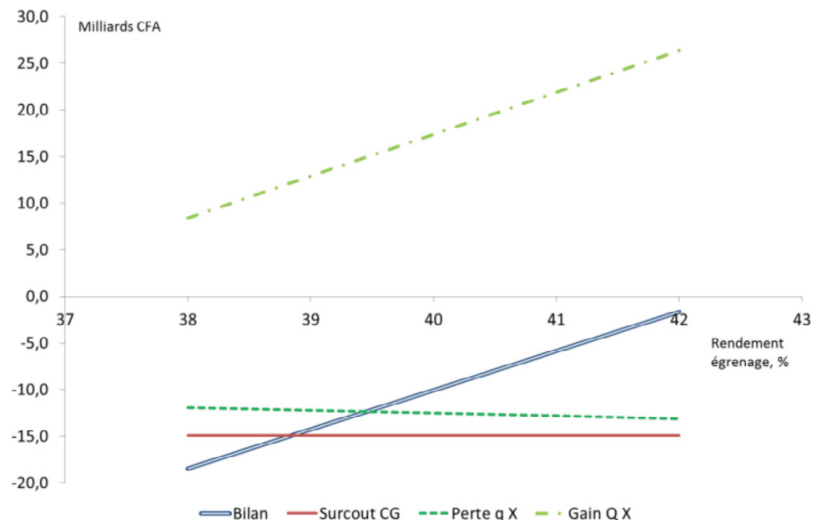

Bilan = bilan financier des sociétés cotonnières; Surcout CG = Montant payé aux producteurs pour le supplément de production obtenue; Perte q X = pertes à l'exportation du fait de l'application d'une décote pour la perte de longueur et l'application d'un malus; Gain Q X = Gain à l'exportation du fait de l'augmentation de la quantité exportée

Box c : Variation du bilan financier de la filière pour une décote de 3,5 cents US/livre de la fibre exportée et selon le prix mondial du coton, rendement égrenage du coton-Bt à 40\% pour la production de 2013

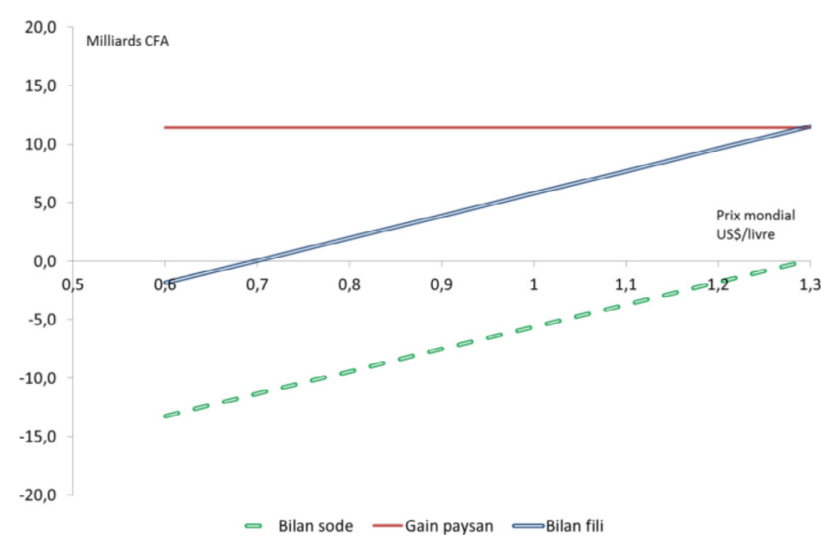

Box $d$ : Variation du bilan financier de la filière pour une décote de 5,0 cents US/livre de la fibre exportée et selon le prix mondial du coton, rendement égrenage du coton-Bt à 40\% pour la production de 2013



Bilan sode = bilan financier des sociétés cotonnières; Gain paysan = Montant perçu par les producteurs en conséquence du supplément de production; Bilan fili $=$ Bilan consolidé des sociétés cotonnières et des paysans

Fig. 2. Variation des bilans financiers des sociétés cotonnières et de la filière selon les niveaux de rendement égrenage des variétés Bt transformées et de prix mondial du coton fibre, pour deux niveaux de décote de prix pour fibre courte (campagne 2013-14).

Fig. 2. Fluctuation of financial balances of cotton companies and of the cotton sector according to ginning outturns of Bt transformed varieties and to world cotton price for two levels of discount applied to lint shortness (campaign 2013-14).

élevée de la décote appliquée à la moindre longueur du coton fibre produit (Fig. 2, Box c et Box d).

Dans l'hypothèse d'un rendement égrenage du coton-Bt de $40 \%$, c'est seulement à partir d'un prix mondial de 1,3 US\$/livre, niveau qu'il est peu probable de trouver dans un proche futur, que le bilan financier des sociétés cotonnières cesse d'être négatif (Fig. 2, Box c). Avec une valeur plus élevée de la décote (Fig. 2, Box d), il faudrait un prix encore plus élevé pour que le bilan devienne positif. Si le prix du marché mondial observé pour la campagne 2013-14 avait correspondu à la tendance historique de ce prix, soit environ 0,8 US\$/livre, le bilan négatif aurait été de plus de 10 milliards FCFA (Fig. 2, Box c) et non pas 7,1 milliards FCFA (Tab. 2).

Le bilan financier consolidé des sociétés cotonnières et des paysans croît avec le niveau du prix mondial mais il 
peut devenir négatif si ce prix est plus bas que celui observé entre les années 2010 et 2013, surtout avec une valeur plus élevée de la décote. Avec une décote de 3,5 cents US/livre, le bilan consolidé est positif à partir d'un prix mondial de 0,70 US\$/livre. En dessous de ce niveau de prix mondial, observé pendant plusieurs années avant l'envolée de 2010, le bilan consolidé est négatif. Avec une valeur de décote de 5,0 cents US/livre, le bilan consolidé est négatif en deçà de 0,85 US\$/livre, ce qui est le cas depuis la fin de l'année 2014 avec un prix mondial en dessous des 0,70 US\$/livre.

\section{Conclusion}

Sur la base d'un scénario considéré comme étant proche de la réalité, les impacts financiers du coton-Bt au Burkina Faso sont décevants et loin de ce qui a été anticipé.

Pour les paysans pris globalement, le bilan est positif à un niveau assez faible et avec un niveau de risque financier peu incitateur. Ce bilan est largement inférieur à ce qui était espéré.

Pour les sociétés cotonnières, le bilan financier est modeste et devenu négatif à mesure que le prix mondial du coton fibre est redescendu de son record historique et s'est rapproché de sa tendance de long terme. Cela explique que les sociétés cotonnières se soient rendu compte assez tardivement du caractère négatif du coton-Bt pour leur bilan financier. Ce caractère négatif s'est accentué avec la poursuite de la baisse du prix mondial; baisse qui devrait durer au regard de la conjoncture économique mondiale.

Le caractère négatif de l'adoption du coton-Bt pour les sociétés cotonnières est la conséquence des pertes industrielle et commerciale découlant d'une mauvaise réalisation de la transformation génétique des variétés africaines fournies. L'on comprend que les sociétés cotonnières réclament des compensations à Monsanto. Le cas étudié apporte aussi une première infirmation de l'hypothèse d'équivalence de produits couramment retenue dans les études d'évaluation d'impacts des OGM en agriculture.

Le coton du Burkina Faso pâtit désormais d'une perte d'image qui demandera du temps à être rétablie. Les pertes constatées peuvent alors durer. Le fait que des pays de la sousrégion aient augmenté leur production tout en conservant l'image de leur coton rend la tâche facile aux négociants pour imposer un malus au Burkina Faso. Une telle conséquence est ironique pour un pays qui a tant misé sur la qualité pour bénéficier de bonus sur le marché mondial.

L'adoption du coton-Bt au Burkina Faso, dans les conditions qui ont prévalu, a correspondu à un cas de changement technologique non Pareto-efficace, avec des impacts divergents entre les deux catégories d'acteurs. Pis, cette divergence peut hypothéquer la collaboration entre ces acteurs dans la poursuite du progrès technique si ce n'est dans le fonctionnement plus global de la filière.

Les résultats observés au Burkina Faso ne sont pas inhérents à l'adoption du coton-Bt en tant que telle, mais la conséquence d'une mauvaise qualité d'exécution de la transformation des variétés découlant d'une certaine précipitation. Tout autre pays tenté par l'aventure de l'adoption du coton-Bt doit s'assurer d'obtenir la qualité nécessaire des variétés transformées à utiliser.

\section{Références}

AFP. 2015. Le Burkina Faso va réduire sa production de coton transgénique à cause de problèmes techniques. AFP 07 mai 15 à 18 h 23. Ouagadougou (Burkina Faso).

Airault P. 2012. Jean Paul Sawadogo : sur les OGM, notre bilan est très positif. Consulté le 21/03/2015 au http://www.jeuneafrique. com/Articleimp_JA2668p084.xml0_jean-paul-sawadogo-sur-lesogm-notre-bilan-est-tr-s-positif.html.

Cabanilla LS, Abdoulaye T, Sanders HJ. 2005. Economic cost of nonadoption of Bt Cotton in West Africa: with special reference to Mali. Int. J. Biotechnol. 7(1/2/3): 46-61.

Dowd-Uribe B. 2014. Engineering yields and inequality? How institutions and agro-ecology shape $\mathrm{Bt}$ cotton outcomes in Burkina Faso. Geoforum 53: 161-171.

Dowd-Uribe BM, Bingen J. 2011. Debating the merits of biotech crop adoption in sub-Saharan Africa: distributional impacts, climatic variability and pest dynamics. Prog. Dev. Stud. 11: 63-68.

Elbehri A, Macdonald S. 2004. Estimating the Impact of Transgenic Bt Cotton on West and Central Africa: A general Equilibrium Approach. World Dev. 32(12): 2049-2064.

Fichet M. 1996. L'impact de la privatisation dans les pays africains de la zone Franc. Coton Dev. 20: 8-18.

Fok M. 2004. Les facteurs d'efficacité des Systèmes de règlements privés comme institutions de régulation des transactions marchandes. In: Premier Colloque de l'Association Française de Sociologie, Villetaneuse (France), 24-27 février 2004, p. 19.

Fok M. 2010. Facteurs d'efficacité des arrangements institutionnels en politique cotonnière africaine. Cah. Agric. 19(1): 68-74.

Gouse M, Pray CE, Schimmelpfennig DE. 2004. The distribution of benefits from Bt Cotton adoption in South Africa. AgBioForum 7(4): 187-194.

Kaminski J, Headey D, Bernard T. 2011. The Burkinabé Cotton Story 1992-2007: Sustainable Success or Sub-Saharan Mirage? World Dev. 39: 1460-1475.

Kuraparthy V, Bowman D. 2013. Gains in Breeding Upland Cotton for Fiber Quality. J. Cotton Sci. 162: 157-162.

Qayum A, Sakkhari K. 2005. Bt cotton in Andhra Pradesh. A Threeyear assessment. The first ever sustained independent scientific study on Bt cotton in India, Zaheerabad: Deccan Development society.

Renaudin C, Pelc H, Opois J. 2012. Cotonnier génétiquement modifié : l'expérience d'une campagne agricole au Burkina Faso. Cah. Agric. 21(6): 387-394.

Sigrist JC. 2014. Plan Coton Génétiquement Modifié : impact agronomique et économique. In: Géocoton Séminaire 2014. Le coton africain : évolution des principaux facteurs de production en zone cotonnière. 29/09-03/10/2014.

Stiller W, Reid P, Constable G. 2006. Lessons learnt in developing transgenic cotton (Gossypium hirsutum) varieties. Breeding for Success: Diversity in Action. In: Proceedings 13th Australasian Plant Breeding Conference. Christchurch, New Zealand. 18-21 April 2006.

Tao A. 2012. Coton-Bt au Burkina Faso. Quotidien Le Pays. Consulté le 12/06/2012 au http://www.nepadbiosafety.net/fr/coton-bt-auburkina-faso.

Traxler G, Godoy-Avila S. 2004. Transgenic cotton in Mexico. AgBioForum 7(1 et 2): 57-62.

Vitale J, Boyer T, Uaiene R, Sanders JH. 2007. The Economic Impacts of Introducing Bt Technology in Smallholder Cotton Production Systems of West Africa: A Case Study from Mali. AgBioForum 10(2): 71-84. 
Vitale J, Glick H, Greenplate J, Abdennadher M, Traoré O. 2008. Second-Generation Bt Cotton Field Trials in Burkina Faso: Analyzing the Potential Benefits to West African Farmers. Crop Sci. 48: $1958-1966$.
Zepeda JBF, Nelson RG. 1997. Rent Creation and Distribution From Biotechnology Innovations: The Case of Bt Cotton and Herbicide-Tolerant Soybeans in 1997. Agribusiness 16(1): 21-32.

Cite this article as: Fok M. 2016. Impacts du coton-Bt sur les bilans financiers des sociétés cotonnières et des paysans au Burkina Faso. Cah. Agric. 25: 35001. 